\title{
Optimizing sequential treatment with anti-EGFR and VEGF mAb in metastatic colorectal cancer: current results and controversies
}

This article was published in the following Dove Medical Press journal: Cancer Management and Research

\author{
Datian Chen' \\ Kaikai $\mathrm{Gu}^{2}$ \\ Huiyu Wang ${ }^{3}$ \\ 'Department of Oncology, Haimen \\ People's Hospital, Haimen, People's \\ Republic of China; ${ }^{2}$ Haimen Hospital \\ of Traditional Chinese Medicine, \\ Haimen, People's Republic of China; \\ ${ }^{3}$ Wuxi People's Hospital Affiliated \\ to Nanjing Medical University, Wuxi, \\ People's Republic of China
}

Correspondence: Huiyu Wang Wuxi People's Hospital Affiliated to Nanjing Medical University, 299 Qingyang Road, Wuxi, People's Republic of China Email wanghuitu20II@I26.com

\begin{abstract}
Anti-EGFR mAb (cetuximab or panitumumab) and anti-VEGF mAb (bevacizumab) are the two main targeted agents available for RAS wild-type (WT) metastatic colorectal cancer (mCRC) treatment. Nonetheless, three head-to-head clinical trials evaluating anti-EGFR mAb vs -VEGF $\mathrm{mAb}$ in first-line treatment failed to conclude a uniform result. Recently, a few small clinical studies revealed that prior use of bevacizumab may impair the effect of cetuximab or panitumumab. Preclinical studies have also suggested that pretreatment with bevacizumab may lead to simultaneous resistance to anti-EGFR mAb. Therefore, we performed this review to summarize the available data regarding the optimal sequential treatment of anti-EGFR and -VEGF mAb for RAS or KRAS WT mCRC and discuss the potential mechanisms that may explain this phenomenon. Primary tumor location and early tumor shrinkage have emerged as new potential prognostic and predictive factors in mCRC. We also collected information to explore whether these factors affect the optimal sequencing of targeted therapy in mCRC. However, definite conclusions cannot be made, and we can only speculate on optimal treatment recommendations based on the contradictory results.
\end{abstract}

Keywords: anti-EGFR mAb, bevacizumab, treatment sequence, metastatic colorectal cancer

\section{Introduction}

Colorectal cancer is a leading cause of cancer-related death worldwide with over 1.8 million new cases and 881,000 deaths in $2018 .{ }^{1}$ However, with improvements in both targeted biological therapy and surgical intervention, median survival has exceeded 30 months in some patients with metastatic colorectal cancer (mCRC) by better understanding tumor biology and abundant treatment experience. ${ }^{2-4}$

EGFR antibodies, including cetuximab and panitumumab, have been widely used in first-line $\mathrm{mCRC}$ treatment, and RAS mutations represent a negative predictive indicator for EGFR antibodies. Therefore, NCCN guidelines now recommend that anti-EGFR mAb should be applied in RAS wild-type (WT) mCRC. ${ }^{5}$ Bevacizumab is an antibody targeted at VEGF-A, and although predictive biomarkers of bevacizumab have not yet been identified, it has improved the first-line therapy efficacy and is often continued in the second-line setting after progression on first-line bevacizumab. ${ }^{6-10}$

Increasing evidence suggests that primary tumor location correlates with distinct molecular and clinical characteristics. Recently, two meta-analyses were performed to investigate the prognostic and predictive effects of primary tumor location based on the first-line clinical trials in unresectable RAS WT mCRC. Right-sided mCRC had worse prognosis than left-sided mCRC. Regarding the predictive effect of primary tumor 
location, patients with left-sided $\mathrm{mCRC}$ had a significant survival benefit from anti-EGFR mAb plus chemotherapy compared to that from bevacizumab plus chemotherapy. ${ }^{11,12}$ In contrast, bevacizumab-based treatment had a numerical survival advantage in patients with right-sided mCRC. However, the potential molecular mechanisms that may contribute to the differential clinical outcomes and responses to therapy behind the tumor sidedness remain unclear. Differences in gut content, epigenetic alterations, genomic instability, consensus molecular subtype classification, and mutation status may explain the phenomenon. ${ }^{13}$

Making all anticancer drugs available to patients with $\mathrm{mCRC}$ is important to achieve the maximal benefit for long-term survival irrespective of the chemotherapy drug sequence. ${ }^{14,15}$ Nevertheless, the optimal use and sequence of targeted therapy is still controversial, especially in $\mathrm{mCRC}$ patients after progression on first-line bevacizumab. In the FIRE-3 study, no difference was observed in progression-free survival (PFS) between first-line cetuximab and bevacizumab biologic therapies, while overall survival (OS) favored the cetuximab group regardless of the KRAS or RAS WT populations. ${ }^{16}$ In contrast, the results from CALGB/SWOG 80405 trial showed there were no significant differences in survival outcomes between the addition of bevacizumab vs cetuximab to first-line chemotherapy. ${ }^{17}$ Additionally, prospective trials produced conflicting results when comparing the second-line efficacy of anti-EGFR mAb vs bevacizumab after progression on first-line bevacizumab. ${ }^{18,19}$

To further explore an optimal treatment sequence of antiEGFR and -VEGF $\mathrm{mAb}$ in $\mathrm{mCRC}$, we conduct this review of the available clinical trial data and observational studies, and discuss potential mechanisms that may explain the contradiction in targeted drug treatment sequence.

\section{Findings}

\section{Head-to-head anti-EGFR vs -VEGF mAb in first-line treatment}

Three randomized clinical trials have investigated the addition of anti-EGFR mAb or bevacizumab to first-line standard chemotherapy in RAS WT mCRC (Table 1). FIRE-3 study compared first-line FOLFIRI plus cetuximab to FOLFIRI plus bevacizumab in patients with initial KRAS WT mCRC. ${ }^{16}$ Extended RAS analysis identified 400 patients with RAS WT mCRC. In the final RAS WT population, the objective response rate (ORR) $(65.3 \%$ vs $58.7 \% ; P=0.18$ for cetuximab vs bevacizumab groups) and PFS (10.3 vs 10.2 months; $P=0.77$ for cetuximab vs bevacizumab groups) were not significantly different between the two treatments. In contrast, cetuximab plus FOLFIRI was associated with significantly longer OS than bevacizumab plus FOLFIRI (33.1 vs 25.0 months; $P=0.0059$ ). Within the 330 RAS WT patients with centralized radiological review, early tumor shrinkage (ETS) was achieved more frequently in the cetuximab + FOLFIRI group than in the bevacizumab + FOLFIRI group (68.2\% vs $49.1 \% ; P=0.0005)$. Likewise, the median depth of response (DpR) was higher in the cetuximab plus FOLFIRI group (48.9\% vs $32.3 \% ; P<00001)$.

Next, the CALGB/SWOG 80405 trial of first-line cetuximab vs bevacizumab combined with either FOLFIRI

Table I Anti-EGFR vs -VEGF mAb in first-line treatment for patients with RAS WT mCRC

\begin{tabular}{|c|c|c|c|c|c|c|}
\hline & $\begin{array}{l}\text { RAS WT } \\
\text { population (n) }\end{array}$ & ETS (\%) & $\begin{array}{l}\text { Median } \\
\text { DpR (\%) }\end{array}$ & ORR (\%) & $\begin{array}{l}\text { Median PFS } \\
\text { months }\end{array}$ & $\begin{array}{l}\text { Median OS } \\
\text { months }\end{array}$ \\
\hline \multicolumn{7}{|l|}{ FIRE-3 $3^{16}$} \\
\hline Cetuximab + FOLFIRI & 199 & 68.2 & 48.9 & 65 & 10.3 & 33.1 \\
\hline Bevacizumab + FOLFIRI & 201 & 49.1 & 32.3 & 58.7 & 10.2 & 25 \\
\hline HR or OR $(95 \% \mathrm{Cl})$ & & $\begin{array}{l}2.22(I .4 I-3.47) \\
P=0.0005\end{array}$ & $\begin{array}{l}\text { NA; } \\
P<0.00 \text { I }\end{array}$ & $\begin{array}{l}1.33(0.88-1.99) \\
P=0.18\end{array}$ & $\begin{array}{l}0.97(0.78-1.20) \\
P=0.77\end{array}$ & $\begin{array}{l}0.70(0.54-0.90) \\
P=0.0059\end{array}$ \\
\hline \multicolumn{7}{|l|}{ CALGB/SWOG $80405^{17,20}$} \\
\hline Cetuximab + chemotherapy & 270 & NR & NR & 69 & 11.2 & 32 \\
\hline Bevacizumab + chemotherapy & 256 & NR & NR & 54 & 11.0 & 31.2 \\
\hline HR or OR $(95 \% \mathrm{Cl})$ & & & & $\begin{array}{l}N R ; \\
P<0.01\end{array}$ & $\begin{array}{l}I .03(0.86-I .24) \\
P=0.7 \mid\end{array}$ & $\begin{array}{l}0.88(0.72-1.08) \\
P=0.24\end{array}$ \\
\hline \multicolumn{7}{|l|}{ PEAK $^{21}$} \\
\hline Panitumumab + FOLFOX & 88 & 64 & 65 & 65 & 12.8 & 36.9 \\
\hline Bevacizumab + FOLFOX & 82 & 45 & 46.3 & 60 & 10.1 & 28.9 \\
\hline HR or OR $(95 \% \mathrm{Cl})$ & & $\begin{array}{l}1.99(0.99-4.10) ; \\
P=0.052\end{array}$ & $\begin{array}{l}\mathrm{NA} ; \\
P=0.0018\end{array}$ & $\begin{array}{l}\text { I. I2 (0.56-2.22); } \\
P=0.86\end{array}$ & $\begin{array}{l}0.68(0.48-0.96) \\
P=0.029\end{array}$ & $\begin{array}{l}0.76(0.53-1.1 \mathrm{I}) ; \\
P=0.15\end{array}$ \\
\hline
\end{tabular}

Note: ETS was defined as having $\geq 30 \%$ tumor shrinkage at week 8 in PEAK and 20\% tumor shrinkage at week 6 in FIRE-3.

Abbreviations: DpR, depth of response; ETS, early tumor shrinkage; mCRC, metastatic colorectal cancer; NA, not available; NR, not reported; ORR, objective response rate; OS, overall survival; PFS, progression-free survival; WT, wild-type. 
or mFOLFOX6 regimen enrolled 526 RAS WT mCRC patients. ${ }^{17,20}$ No differences in survival outcomes were observed in this cohort. The median OS was 32 months in the cetuximab group and 31.2 months in the bevacizumab group (HR: $0.88 ; P=0.24$ ). The median PFS was 11.2 months in the cetuximab group and 11.0 months in the bevacizumab group (HR: $1.03 ; P=0.71)$. ORR was also higher in the cetuximab arm $(69 \%$ vs $54 \% ; P<0.01)$. Finally, the PEAK trial compared panitumumab plus mFOLFOX6 to bevacizumab plus mFOLFOX6 for the first-line treatment of $\mathrm{mCRC}$ in an RAS WT population. ${ }^{21}$ Median DpR was improved in the panitumumab + mFOLFOX6 arm compared to that in bevacizumab + mFOLFOX6 (65.0\% vs 46.3\%; $P=0.0018$ ). More patients experienced ETS in the panitumumab group (64\% vs $45 \%$; $P=0.052)$. No significant differences in ORR were observed between the two groups ( $65 \%$ vs $60 \% ; P=0.86)$. However, PFS was significantly prolonged in the panitumumab group (12.8 vs 10.1 months; $P=0.029$ ), and OS was numerically longer for the panitumumab group vs the bevacizumab group (36.9 vs 28.9 months; $P=0.15$ ).

FIRE-3 and PEAK also explored the survival outcomes according to the ETS. For patients achieving ETS, anti-EGFR $\mathrm{mAb}$ numerically prolonged OS compared to bevacizumab in both studies (OS: 38.3 vs 31.9 months, $P=0.48$ in FIRE3; 43.8 vs 35.1 months, $P=0.41$ in PEAK). ${ }^{16,21}$ Among the patients without ETS, a markedly shorter OS was observed, which was comparable between the anti-EGFR mAb and bevacizumab groups ( 20.5 vs 21.2 months, $P=0.59$ in FIRE-3; 34.2 vs 23.9 months, $P=0.31$ in PEAK). The predictive effects of tumor location have been reported in a previous article. ${ }^{12}$ In the PEAK study, panitumumab plus mFOLFOX6 appears to be numerically superior to bevacizumab plus mFOLFOX6 in median PFS (14.6 vs 11.5 months; $P=0.07$ ) and median OS (43.4 vs 32.0 months; $P=0.31$ ) for patients with left-sided tumors. Bevacizumab plus mFOLFOX6 increases median PFS (12.6 vs 8.7 months; $P=0.91)$ and median OS (21.04 vs 17.4 months; $P=0.32$ ) in patients with right-sided mCRC compared to panitumumab plus mFOLFOX6. Regarding the cetuximab trials, tumor sidedness was also associated with different outcomes. In the FIRE-3 trial, patients treated with cetuximab plus FOLFIRI significantly benefited in terms of OS compared to patients treated with FOLFIRI plus bevacizumab in left-sided RAS WT mCRC, while no obvious differences were observed in right-sided tumors between the two arms. Cetuximab and bevacizumab also have different treatment efficacies according to the tumor location with cetuximab performing better in left-sided tumors and with bevacizumab performing better in right-sided tumors in the CALGB 80405 trial.

Additionally, the predictive role of ETS based on primary tumor sidedness was evaluated in the FIRE-3 and PEAK trials (Table 2). FIRE-3 used centralized radiological

Table 2 ETS and outcomes according to the primary tumor location

\begin{tabular}{|c|c|c|c|c|}
\hline Trials & \multicolumn{2}{|c|}{ Treatment arms } & $\begin{array}{l}\text { Median PFS (months), } \\
\text { HR }(95 \% \mathrm{Cl})\end{array}$ & $\begin{array}{l}\text { Median OS (months), } \\
\text { HR }(95 \% \mathrm{Cl})\end{array}$ \\
\hline \multicolumn{5}{|c|}{ Left-sided colorectal cancer } \\
\hline FIRE-322 & $\begin{array}{l}\text { FOLFIRI + cet } \\
\text { FOLFIRI + bev }\end{array}$ & \multirow[t]{2}{*}{$\begin{array}{l}\text { ETS } \geq 20 \% \\
(30 \%)\end{array}$} & NR & NR \\
\hline PEAK $^{23}$ & $\begin{array}{l}\text { FOLFOX + pani } \\
\text { FOLFOX + bev }\end{array}$ & & 16.2 vs 12.9 months & 55.4 vs 48.5 months \\
\hline FIRE-3 & $\begin{array}{l}\text { FOLFIRI + cet } \\
\text { FOLFIRI + bev }\end{array}$ & \multirow[t]{2}{*}{$\begin{array}{l}\text { ETS }<20 \% \\
(30 \%)\end{array}$} & NR & NR \\
\hline PEAK & $\begin{array}{l}\text { FOLFOX + pani } \\
\text { FOLFOX + bev }\end{array}$ & & II.6 vs 12.4 months & 34.2 vs 27.7 months \\
\hline \multicolumn{5}{|c|}{ Right-sided colorectal cancer } \\
\hline FIRE-3 & $\begin{array}{l}\text { FOLFIRI + cet } \\
\text { FOLFIRI + bev }\end{array}$ & \multirow[t]{2}{*}{$\begin{array}{l}\text { ETS } \geq 20 \% \\
(30 \%)\end{array}$} & $\begin{array}{l}7.8 \text { vs } 13.4 \text { months } \\
1.7 \mid 8(0.83-3.55) P=0.137\end{array}$ & $\begin{array}{l}27.9 \text { vs } 23.2 \text { months } \\
1.054(0.45-2.45) P=0.903\end{array}$ \\
\hline PEAK & $\begin{array}{l}\text { FOLFOX + pani } \\
\text { FOLFOX + bev }\end{array}$ & & 10.8 vs 18.4 months & 24.6 vs 26.2 months \\
\hline FIRE-3 & $\begin{array}{l}\text { FOLFIRI + cet } \\
\text { FOLFIRI + bev }\end{array}$ & \multirow[t]{2}{*}{$\begin{array}{l}\text { ETS }<20 \% \\
(30 \%)\end{array}$} & $\begin{array}{l}2.8 \text { vs } 5.2 \text { months } \\
1.743(0.84-3.61) \mathrm{P}=0.129\end{array}$ & $\begin{array}{l}\text { II.7 vs } 15.9 \text { months } \\
1.902(0.89-4.06) \mathrm{P}=0.0902\end{array}$ \\
\hline PEAK & $\begin{array}{l}\text { FOLFOX + pani } \\
\text { FOLFOX + bev }\end{array}$ & & 5.8 vs 12.6 months & I5.3 vs 23.3 months \\
\hline
\end{tabular}

Notes: (30\%) represent percentage value for PEAK trial. ETS was defined as having $\geq 30 \%$ tumor shrinkage at week 8 in PEAK and $20 \%$ tumor shrinkage at week 6 in FIRE-3. Abbreviations: bev, bevacizumab; cet, cetuximab; ETS, early tumor shrinkage; NR, not reported; OS, overall survival; pani, panitumumab; PFS, progression-free survival. 
review data including 325 RAS WT mCRC patients to calculate ETS and DpR. ${ }^{22}$ In patients with right-sided tumors achieving ETS, the addition of cetuximab to FOLFIRI had comparable treatment efficacy compared to bevacizumab to FOLFIRI (OS: 27.9 vs 23.2 months, $P=0.90$; PFS: 7.8 vs 13.4 months, $P=0.14)$. In patients with right-sided tumors not achieving ETS, a markedly inferior survival outcome was observed in the cetuximab group compared to that in the bevacizumab group (OS: 11.7 vs 15.9 months, $P=0.09$; PFS: 2.8 vs 5.2 months, $P=0.13$ ). In PEAK, a greater proportion of patients experienced ETS and DpR with panitumumab than bevacizumab irrespective of the primary tumor location. ${ }^{23}$ Panitumumab improved median OS in patients with left-sided mCRC regardless of achieving ETS or not. Among patients with right-sided mCRC, median OS was also comparable between the panitumumab and bevacizumab arms in those patients achieving ETS (OS: 24.6 vs 26.2 months for the panitumumab vs bevacizumab groups). Therefore, patients with right-sided mCRC achieving ETS may benefit from an anti-EGFR mAb.

\section{Conflicting results from retrospective studies in $\mathrm{mCRC}$}

In 2011, Norguet et al found that anti-VEGF pretreated patients had a lack of response to cetuximab and a poorer decreased disease-specific survival compared to the antiVEGF naïve patients (4.9 vs 9.1 months; $P=0.026$ ). The results remained statistically significant after adjusting for KRAS status in the multivariate Cox model. ${ }^{24}$ Similarly, another study reported that anti-EGFR treatment had a poorer PFS in the pretreatment with bevacizumab-based chemotherapy group compared to the chemotherapy alone as first-line therapy ( 2.8 vs 4 months; $P=0.003$ ), but the OS was not significantly different in the two groups. ${ }^{25}$ Moreover, Sato et al showed that cetuximab significantly improved PFS in patients with no prior bevacizumab use than in patients with prior bevacizumab use in univariate analysis $(P=0.048$ for second-line cetuximab-based therapy; $P=0.0022$ for third-line cetuximab-based therapy). Importantly, they additionally found that a shorter PFS was observed in the group of $<3$ months from last bevacizumab use to third-line cetuximab therapy. ${ }^{26}$ Consistent with this observation, a cohort study revealed that a short-time interval between the last bevacizumab administration and the anti-EGFR therapy attenuates the efficacy of anti-EGFR mAb in KRAS WT mCRC patients. ${ }^{27}$ However, in a subgroup analysis of the
20050181 study, patients receiving prior oxaliplatin-bevacizumab also benefited from panitumumab plus FOLFIRI compared to those receiving FOLFIRI alone. ${ }^{28}$ Alternatively, a small retrospective analysis that investigated the survival of KRAS WT patients who were sequentially treated with cetuximab and bevacizumab reported that patients receiving bevacizumab first had similar survival outcomes compared to patients receiving cetuximab first (PFS: 13 vs 10 months, $P=0.798$; OS: 44 vs 39 months, $P=0.862) .{ }^{29}$ Burge et al analyzed a multicenter registry and found that initial bevacizumab use did not impact the efficacy of EGFR antibodies in subsequent therapy as PFS and OS from anti-EGFR mAb commencement were not significantly different from prior bevacizumab use, ${ }^{32}$ which is similar to other reports. ${ }^{30,31}$ In addition, a longer gap between bevacizumab and anti-EGFR $\mathrm{mAb}$ (>6 months) was linked to a longer median PFS in right-sided tumors, while left-sided tumors had no difference, which may reflect a distinct subgroup with a superior prognosis in right-sided tumors. ${ }^{32}$

There are two retrospective analyses from clinical trials that explore the efficacy of subsequent treatment. ${ }^{33,34}$ In the FIRE-3 study, the study protocol recommended FOLFOX plus bevacizumab as second-line regimen in the initial FOLFIRI plus cetuximab group and irinotecan plus cetuximab in the initial FOLFIRI plus bevacizumab group. Second-line PFS and OS were significantly improved in the initial FOLFIRI plus cetuximab arm compared to the initial FOLFIRI plus bevacizumab arm in the original KRAS WT populations (second PFS: 6.5 vs 4.7 months, $P<0.001$; second OS: 16.3 vs 13.2 months, $P=0.0021$ ). Upon assessment of expanded RAS WT populations, the treatment effects (second PFS: 6.7 vs 4.8 months, $P=0.003$, second OS; 17.6 vs 14.8 months, $P=0.0021$ ) were even more pronounced in the initial cetuximab plus FOLFIRI group vs bevacizumab plus FOLFIRI group. The post hoc analysis also investigated the sequence of cetuximab and bevacizumab or the reverse sequence according to the primary tumor sidedness. A more favorable second-line PFS and OS of cetuximab followed by bevacizumab was observed in patients with left-sided mCRC compared to that with the reverse sequence (second PFS: 7.3 vs 5.8 months, $P=0.005$; second OS: 15.9 vs 9.7 months, $P=0.007$ ). However, in patients with right-sided $\mathrm{mCRC}$, no benefit was observed in either defined sequences.

Another study evaluated the OS for patients treated with first-line panitumumab and then the second-line bevacizumab 
or the reverse sequence in a total of the PEAK, PRIME, and 20050181 trials. ${ }^{35}$ A pooled analyses of OS suggested a benefit from the panitumumab and then bevacizumab treatment sequence compared to bevacizumab followed by panitumumab in RAS WT mCRC (median OS: 36.8 vs 27.8 months; $P=0.06)$. The median OS was further prolonged in patients with left-sided tumors ( 43.4 vs 32.4 months; $P=0.10$ ) and RAS/BRAF WT mCRC (41.3 vs 28.9 months; $P=0.03$ ).

Taken together, although these results were not consistent, prior use of bevacizumab may affect the efficacy of cetuximab or panitumumab. Nevertheless, the role of primary tumor location in treatment sequence is less clear.

\section{Anti-EGFR $m A b$ vs bevacizumab after progression on first-line bevacizumab}

Importantly, studies have now begun to explore anti-EGFR $m A b$ vs bevacizumab in patients with KRAS WT mCRC after progression on first-line bevacizumab. Table 3 summarizes the available evidence from three clinical trials.

The SPIRITT study compared FOLFIRI plus panitumumab to FOLFIRI plus bevacizumab as second-line treatment in patients with KRAS WT mCRC after failure of first-line oxaliplatin-based chemotherapy regimen containing bevacizumab. ${ }^{18}$ The trial enrolled a total of 182 patients with KRAS WT mCRC. Patients in the bevacizumab + FOLFIRI arm had longer median PFS and OS than those in the panitumumab + FOLFIRI arm (PFS: 9.2 vs 7.7 months, $P=0.97$; OS: 21.4 vs 18.0 months, $P=0.75)$. The panitumumab group had a higher ORR than the bevacizumab group (32\% vs $19 \%$ ). Safety data indicated higher rates of Grade $>3$ AEs in the panitumumab group compared to that in the bevacizumab group ( $85 \%$ vs $75 \%$, respectively). Skin disorders and diarrhea were more frequent in the panitumumab group, while neutropenia was more frequent in the bevacizumab group. The main limitations include two aspects. First, there was an imbalance in subsequent therapies between the two groups. Subsequent anti-EGFR (54\% vs 26\%) and anti-VEGF (24\% vs $20 \%$ ) therapies were both more frequent in the bevacizumab arm compared to that in the panitumumab arm. Second, patients in the panitumumab group were older and had more metastatic sites. Because these factors can potentially affect OS, we should interpret these results with caution.

Another study, WJOG 6210G, also investigated the second-line treatment of panitumumab plus FOLFIRI vs bevacizumab plus FOLFIRI in KRAS WT mCRC after progression on first-line bevacizumab-based chemotherapy. ${ }^{36}$ One hundred and seventeen patients were eligible, and patient characteristics between the two groups were well balanced. Median OS was 16.2 months for panitumumab plus FOLFIRI and 13.4 months for bevacizumab plus FOLFIRI (HR: 1.16; 95\% CI: 0.76-1.77). PFS was 6.0 months for panitumumab plus FOLFIRI and 5.9 months for bevacizumab plus FOLFIRI (HR: 1.14; 95\% CI: 0.78-1.66). The ORR was higher in the panitumumab group $(46.2 \%$ vs $5.7 \% ; P<0.001)$. Overall, the two therapeutic regimens had similar efficacy. Interestingly, RAS and BRAF mutation analysis showed

Table 3 Anti-EGFR vs -VEGF mAb in second-line treatment after progression on first-line bevacizumab for patients with KRAS WT $\mathrm{mCRC}$

\begin{tabular}{|c|c|c|c|c|c|}
\hline & $\begin{array}{l}\text { Primary end- } \\
\text { point }\end{array}$ & $\begin{array}{l}\text { KRAS WT } \\
\text { population }\end{array}$ & & & \\
\hline & & $\mathbf{n}$ & ORR (\%) & $\begin{array}{l}\text { Median PFS } \\
\text { months }\end{array}$ & $\begin{array}{l}\text { Median OS } \\
\text { months }\end{array}$ \\
\hline SPIRITT'18 & OS & & & & \\
\hline Panitumumab + FOLFIRI & & 91 & 32 & 7.7 & 18 \\
\hline Bevacizumab + FOLFIRI & & 91 & 19 & 9.2 & 21.4 \\
\hline HR or OR $(95 \% \mathrm{Cl})$ & & & NR & $\begin{array}{l}\text { I.0I (0.68-I.50); } \\
P=0.97\end{array}$ & $\begin{array}{l}\text { I.06 (0.75-I.49); } \\
P=0.75\end{array}$ \\
\hline WJOG6210G 36 & ORR & & & & \\
\hline Panitumumab + FOLFIRI & & 59 & 46.20 & 6 & 16.2 \\
\hline Bevacizumab + FOLFIRI & & 58 & 5.70 & 5.9 & 13.4 \\
\hline HR or OR $(95 \% \mathrm{Cl})$ & & & $N R ; P<0.00 I$ & $1.14(0.78-1.66)$ & $1.16(0.76-1.77)$ \\
\hline PRODIGE-I $18^{19}$ & PFS & & & & \\
\hline Bevacizumab + chemotherapy & & 65 & NR & 7.1 & 15.8 \\
\hline Cetuximab + chemotherapy & & 67 & NR & 5.6 & 10.4 \\
\hline HR or OR $(95 \% \mathrm{Cl})$ & & & NR & $\begin{array}{l}0.7 \mid(0.50-I .02) ; \\
P=0.06\end{array}$ & $\begin{array}{l}0.69(0.46-1.04) ; \\
P=0.08\end{array}$ \\
\hline
\end{tabular}

Abbreviations: mCRC, metastatic colorectal cancer; NR, not reported; OS, overall survival; ORR, objective response rate; PFS, progression-free survival; WT, wild-type. 
that panitumumab plus FOLFIRI had favorable survival in RAS/BRAF WT patients whereas unfavorable survival in patients with RAS or BRAF mutation compared to bevacizumab plus FOLFIRI. Serum protein analysis suggested that bevacizumab plus FOLFIRI was associated with better OS in the high VEGF-A subgroup and worse OS in patients with low VEGF-A level.

Notably, the phase II PRODIGE-18 study continued to explore the optimized targeted therapy after progression on first-line bevacizumab comparing cetuximab + chemotherapy vs bevacizumab + chemotherapy in KRAS WT mCRC patients. ${ }^{19}$ Median PFS (7.1 vs 5.6 months; $P=0.0622$ ) and OS (15.8 vs 10.4 months; $P=0.0750$ ) were numerically longer in the bevacizumab group than in the cetuximab group. The choice for chemotherapy was based on the first-line regimen (crossover). Upon assessment of expanded RAS WT patients, the bevacizumab-induced effects were more pronounced. The median OS (21.0 vs 10.7 months; $P=0.324$ ) and median PFS ( 7.8 vs 5.6 months; $P=0.076$ ) were both significantly longer in the bevacizumab plus chemotherapy arm vs the cetuximab plus chemotherapy arm.

\section{Different sequence strategy for anti- EGFR mAb after progression on first-line bevacizumab}

Few studies have evaluated the different treatment sequence for anti-EGFR mAb in mCRC patients progressing after first-line chemotherapy/bevacizumab (Table 4). In the phase
III COMETS trial, 53 patients were assigned to receive second-line cetuximab plus irinotecan followed by third-line FOLFOX (arm A) and 55 patients were assigned to receive second-line FOLFOX followed by third-line cetuximab plus irinotecan (arm B). All of the patients were confirmed with KRAS WT tumors. ${ }^{37}$ Median PFS was similar between the two arms (9.9 vs 11.3 months in arm A vs arm B; $P=0.854$ ), while median OS favored arm B compared to arm A (18.6 vs 12.3 months; $P=0.411)$. ORR was higher in arm $B$ than in arm A during the second-line treatment ( $40 \%$ vs $29 \% ; P=0.228$ ), while ORR was similar during the third-line treatment $(21 \%$ vs $23 \% ; P=0.78)$. PFS and OS were also analyzed according to the primary tumor location. In right-sided tumors, median PFS (10.0 vs 7.9 months; $P=0.012)$ and OS (12.6 vs 8.8 months; $P=0.002$ ) were significantly improved in arm $\mathrm{B}$ compared to than in arm A. In left-sided tumors, there was also a trend toward better survival in arm B (median OS: 20.2 vs 13.1 months; $P=0.859$ ).

Also, another randomized phase II REVERSE trial investigated regorafenib followed by cetuximab (R-C arm) vs the reverse sequence ( $\mathrm{C}-\mathrm{R}$ arm) in KRAS WT mCRC patients after progression on first-line chemotherapy. ${ }^{38}$ In total, $96 \%$ of patients in the R-C arm had prior use of bevacizumab in first-line treatment, $98 \%$ did the C-R arm, and $86 \%$ of patients received sequential treatment in both arms. OS was significantly improved in the R-C arm compared to that in the $\mathrm{C}-\mathrm{R}$ arm. PFS I was 2.4 months in the $\mathrm{R}-\mathrm{C}$ arm and 4.2 months in the $\mathrm{C}-\mathrm{R}$ arm $(P=0.91)$. PFS II was 5.2 months in

Table 4 Different sequence strategy for anti-EGFR mAb after progression on first-line bevacizumab

\begin{tabular}{|c|c|c|c|c|c|c|c|}
\hline Trial & Treatment arms & n & $\begin{array}{l}\text { Prior } \\
\text { bevacizumab (\%) }\end{array}$ & PFS II months & PFS III months & $\begin{array}{l}\text { Median PFS } \\
\text { months }\end{array}$ & $\begin{array}{l}\text { Median OS } \\
\text { months }\end{array}$ \\
\hline \multirow[t]{3}{*}{ COMET $^{37}$} & $\begin{array}{l}\text { FOLFOX } 4 \text { followed by } \\
\text { cetuximab + irinotecan }\end{array}$ & 55 & 100 & 6.1 & 4.7 & 11.3 & 18.6 \\
\hline & $\begin{array}{l}\text { Cetuximab + irinotecan } \\
\text { followed by FOLFOX } 4\end{array}$ & 53 & 100 & 5.3 & 4 & 9.9 & 12.3 \\
\hline & HR or OR $(95 \% \mathrm{Cl})$ & & & $\begin{array}{l}0.97(0.65-\mid .45) \\
P=0.88 \mid\end{array}$ & $\begin{array}{l}I .00(0.58-I .70) \\
P=0.986\end{array}$ & $\begin{array}{l}\text { I.04 (0.69-I.56); } \\
P=0.854\end{array}$ & $\begin{array}{l}0.84(0.55-\mid .28) ; \\
P=0.4 \mid I 4\end{array}$ \\
\hline \multirow[t]{3}{*}{ REVERSE $^{38}$} & $\begin{array}{l}\text { Regorafenib followed by } \\
\text { cetuximab + irinotecan }\end{array}$ & 51 & 96 & 2.4 & 5.2 & NR & 17.4 \\
\hline & $\begin{array}{l}\text { Cetuximab }+ \text { irinotecan } \\
\text { followed by regorafenib }\end{array}$ & 50 & 98 & 4.2 & 1.8 & NR & 11.6 \\
\hline & HR or OR $(95 \% \mathrm{Cl})$ & & & $\begin{array}{l}0.97(0.62-1.54) \\
P=0.91\end{array}$ & $\begin{array}{l}0.29(0.17-0.50) \\
P<0.000 \text { I }\end{array}$ & & $\begin{array}{l}0.61(0.39-0.96) ; \\
P=0.029\end{array}$ \\
\hline
\end{tabular}

Notes: PFS was defined as the time from the date of randomization up to the date of progression after third-line treatment or death from any cause. PFS II was defined as the time from the date of randomization up to the date of first progression after second-line or death from any cause. PFS III was defined as the time from the third-line starting date up to the date of first progression after third-line or death from any cause. In REVERSE trial, PFS II was defined as the time from the date of randomization up to the date of first progression after first treatment ( $R$ in R-C arm or C in C-R arm) or death from any cause. PFS III was defined as the time interval between the first progression after first treatment and second progression after second treatment ( $C$ in $R-C$ arm or $R$ in $C-R$ arm).

Abbreviations: OS, overall survival; PFS, progression-free survival. 
the $\mathrm{R}-\mathrm{C}$ arm and 4.2 months in the $\mathrm{C}-\mathrm{R}$ arm $(P<0.0001)$. No unexpected adverse events were observed between the two arms. Subgroup analysis also found that OS was significantly longer in the R-C arm for left-sided tumors (20.5 vs 11.9 months; HR: 0.51 ; 95\% CI: $0.30-0.86 ; P=0.011)$, whereas the results for right-sided tumors were less clear (HR: 0.88 ; 95\% CI: 0.32-2.40). A similar result was observed in RAS/ BRAF WT tumors with better survival in the R-C arm.

\section{Potential mechanisms that explain the puzzling phenomenon Hypoxia-induced resistance to anti-EGFR $\mathrm{mAb}$}

Pàez-Ribes et al reported that therapeutically efficacious antiangiogenic therapy leads to tumor adaptation with heightened invasiveness and enhanced distant metastasis after prolonged treatment in animal models. ${ }^{39}$ A possible reason for these observations may be that the hypoxia/HIFs pathway is an instigator of invasion and metastasis. It has been reported that hypoxia induces angiogenesis, genetic instability, cell survival, epithelial-mesenchymal transition (EMT), altered metabolic pathways, and drug resistance; and HIFs play critical roles therein. ${ }^{40-42}$ One study reported that bevacizumab-resistant mCRC cells expressed higher levels of VEGF and are associated with an intrinsic upregulation of autocrine HIF-VEGF signaling. ${ }^{43}$ Another study revealed an increase in VEGF-A serum levels in $\mathrm{mCRC}$ patients treated with bevacizumab and showed that VEGF-A confers resistance to cetuximab through VEGFR-2/Stat-3 activation. ${ }^{25}$ Therefore, increasing evidence has demonstrated that prior anti-VEGF therapy may contribute to resistance to anti-EGFR $\mathrm{mAb}$ via the hypoxia/HIFs pathway.

Several studies have reported that hypoxia upregulates EGFR expression and immunohistochemical studies showed a close association between EGFR and HIF-1 $\alpha .^{44,45}$ Hypoxia can mediate a proliferative response through an EGF/EGFR autocrine signaling loop in HeLa cells. ${ }^{46}$ Both HIF- $1 \alpha$ and HIF- $2 \alpha$ can trigger the activation of EGFR and downstream targets. ${ }^{47,48}$ Interestingly, HIFs are also EGFR downstream targets and subsequently induce CXCR4, c-MET, VEGF, and survivin to promote EMT, invasion, and metastatic, angiogenic, survival, and anti-apoptotic effects. ${ }^{49}$ Of note, Wang et al also found that hypoxia may play a critical role in resistance to anti-EGFR therapy via HIF-1 $\alpha$ in colorectal cancer. ${ }^{50}$ In addition to HIFs, hypoxia-induced ROS also upregulated the SRC/KRAS/AKT signaling pathway and mediated VEGF augmentation and resistance to apoptosis in colon cells. ${ }^{51}$
The most direct evidence came from the reverse translational research using xenografts of human RAS WT mCRC to explore the biologic rationale underlying the improved efficacy of panitumumab followed by subsequent use of bevacizumab compared to that of the opposite drug sequence. ${ }^{52}$ The panitumumab and then bevacizumab $(\mathrm{PB})$ group was more effective than the bevacizumab and then panitumumab (BP) group in terms of tumor growth inhibition via growth rate and reducing the Ki-67 index. Additionally, phosphoproteomic analysis demonstrated that $\mathrm{PB}$ more efficiently reduced the phosphorylation level of EPHA2 and EGFR. Western blotting confirmed the reduction of EPHA2 protein expression and EPHA2 S897-phosphorylation by PB. RSK phosphorylation was significantly increased with BP but largely unaffected by PB. Moreover, PCR analysis demonstrated statistically significant reductions of lipogenic genes $(F A S N, M V D)$ in the PB group. Considering these results, the hypoxic tumor microenvironment is likely involved because PB significantly suppressed hypoxia-related gene expression (CA9, TGFBI). Treatment with bevacizumab prior to panitumumab created a hypoxic tumor microenvironment that may impair the functions of panitumumab suppressing EGFR downstream signaling and activating the RSK-EphA2 axis and lipid metabolism pathways. So, these results suggest that hypoxic activation of signaling pathways may negatively influence the efficacy of EGFR-targeted therapies and further studies are warranted to elucidate the mechanistic and therapeutic significance in mCRC.

Multi-tyrosine kinase inhibitors (TKIs), such as regorafenib with antiangiogenic activity, can block VEGFR, FGFR, and PDGFR activation and have been approved for mCRC therapy based on a randomized clinical study. ${ }^{53}$ Some scholars posited that judicious doses of anti-VEGF agents could prune abnormal tumor vessels and result in a normalized vasculature, thus alleviating hypoxia via mopping up the excess VEGF, and the clinical data also confirmed that blocking VEGF could normalize tumor vasculature. ${ }^{41,54,55}$ However, tumors can activate other proangiogenic pathways leading to abnormal vessels resulting in hypoxia. Theoretically, TKIs are more likely to restore the abnormal balance between pro-growth and antigrowth factors that lead to tumor vascular abnormality compared to anti-VEGF agents that block a single pro-angiogenic factor because they target several angiogenic axes. Navarro et al reported that TKIs normalized the vasculature and thus corrected hypoxia while an anti-VEGF $m A b$ progressively increased vessel abnormality and hypoxia. ${ }^{56}$ Furthermore, the tumors downregulate aerobic glycolysis and switch to a long-term mitochondrial 
metabolism mediated by downregulation of AKT and HIF-1a and upregulation of PPAR, allowing the use of ketone bodies and fatty acids. These findings demonstrated that acquired resistance to regorafenib may be linked with corrected hypoxia and aberrant cancer metabolism. This may explain the result in the REVERSE trial that regorafenib modified the hypoxic microenvironment leading to R-C sequence efficacy while chemotherapy may cause apoptotic death of tumor cells in the hypoxic tumor areas to create a more favorable condition for anti-EGFR mAb in the COMET trial.

\section{Superior ETS and DPR in first-line anti- EGFR mAb}

Three head-to-head first-line trials, phase III FIRE-3, phase II PEAK, and phase III CALGB 80405 comparing cetuximab or panitumumab to bevacizumab when combined with chemotherapy did not meet their primary endpoints (ORR, PFS or OS, respectively), especially the improved OS in the absence of the differences in ORR or PFS from FIRE-3. ${ }^{57}$ Two recent meta-analyses including these trials demonstrated a significant benefit from an anti-EGFR agent in terms of ORR and OS but not PFS compared to an anti-VEGF agent in RAS WT patients. ${ }^{58,59}$ Therefore, these results have raised questions about how valid these parameters are as adequate surrogate endpoints for OS. Alternatively, ORR according to RECIST failed to capture the temporal and quantitative alterations in tumor burden. ${ }^{60}$ Therefore, two novel response-related endpoints have been utilized in recent trials. ETS, defined as a $20 \%-30 \%$ reduction at $6-8$ weeks, is a predictor for outcomes. It has been reported to be significantly correlated with PFS and OS across multiple trials. ${ }^{61-64} \mathrm{~A}$ meta-analysis demonstrated that ETS has a strong association with survival outcomes, independent of the administered treatment. ${ }^{65}$ Another measurable indicator is depth of response, which assesses the maximum percent change of tumor shrinkage compared to that in baseline. So, the new concepts of ETS and DpR offer a new profile that links new metrics of tumor dynamics with OS to aid clinicians in optimizing mCRC patient management. ${ }^{66}$

Retrospective analyses of the OPUS and CRYSTAL trials revealed that the addition of cetuximab to chemotherapy increased the frequency of ETS and DpR compared to chemotherapy alone in mCRC patients with KRAS WT tumors, which is consistent with the results from FIRE-3. The addition of cetuximab to chemotherapy increased at a greater rate of ETS from $46 \%$ to $69 \%$ and higher median DpR compared to chemotherapy alone (median DpR: $57.9 \%$ vs $30.7 \%$; $P=0.0008)$ in the OPUS trial. Regarding the CRYSTAL study, the frequency of ETS improved from $49 \%$ to $62 \%$ and median DpR increased from $33.3 \%$ to $50.9 \%$. Within both arms in the OPUS and CRYSTAL trials, markedly prolonged PFS and OS were observed in ETS responders compared to that in ETS nonresponders. ETS was linked with improved long-term survival outcomes in patients with mCRC, supporting its potential role as an early predictor of sensitivity to treatment. ${ }^{67,68}$ Furthermore, ETS correlates significantly with depth of response, both of which are significantly linked with post-progression survival and OS. Exploratory analyses from three randomized first-line panitumumab trials also suggested that panitumumab plus chemotherapy was linked with ETS and $\mathrm{DpR}$ benefits compared to chemotherapy alone or chemotherapy plus bevacizumab in RAS WT mCRC patients. ${ }^{69}$ Achieving these endpoints were associated with favorable outcomes, especially for patients with the chance to receive potentially curative resection. Similar results can be observed in the TRIBE trial investigating the efficacy of the addition of oxaliplatin to bevacizumab plus FOLFIRI with higher ETS and DpR in the FOLFOXIRI + bevacizumab arm compared to FOLFIRI + bevacizumab. ${ }^{70,71}$

Taken together, these data support the notion that antiEGFR $\mathrm{mAb}$ has an advantage in tumor shrinkage in temporal and quantitative alterations and provides an explanation for the optimal treatment sequence for anti-EGFR and -VEGF $\mathrm{mAb}$ in $\mathrm{mCRC}$ patients. The first-line treatment effect can translate into tumor shrinkage at nadir. The greatest DpR achieved in first-line treatment can impact the post-progression survival and later lines of therapies are less effective and cannot compensate for the inadequate first-line treatment. ETS is closely correlated with DpR and provides prognostic information. Therefore, it may be hypothesized that the tumor shrinkage benefits from anti-EGFR mAb, and can persistently influence the post-progression survival and OS despite no obvious difference in PFS being observed.

\section{Genome instability in tumor cells and stability in endothelial cells}

Although dramatic genome alteration discrepancy is presented in different tumor types, the evidence from genome repair defects, destabilization of nucleotide sequences, and gene copy number demonstrates that genome instability is an inherent characteristic. ${ }^{72}$ Genome instability provides tumor cells with the ability to adapt to changes in the microenvironment and certain mutant genotypes lead to primary and acquired resistance to anti-EGFR $\mathrm{mAb}$. Therefore, the tumor can spontaneously develop resistant clones that impair the efficacy of cetuximab or panitumumab during tumor progression. ${ }^{73}$ 
Indeed, the second-line 181 trial compared panitumumab plus FOLFIRI to FOLFIRI alone and failed to demonstrate a survival benefit in KRAS WT mCRC with the addition of panitumumab to chemotherapy. ${ }^{28}$ Similarly, in the second-line PICCOLO trials, no significant difference in terms of OS was detected in KRAS WT mCRC patients treated with or without panitumumab. ${ }^{74}$ Alternatively, a preclinical study revealed that the acquisition of KRAS mutations is detectable in the blood as early as 10 months before disease progression through radiological evaluation. ${ }^{75}$ These results suggest that EGFR antibodies should be used as early as possible.

In contrast to tumor cells, endothelial cells have genome stability and theoretically less drug resistance compared to directly targeted tumor cells. ${ }^{76}$ Unlike the mutational mechanisms caused by chemotherapeutics, resistance to bevacizumab might depend on alternative angiogenesis pathways rather than genetic mutations or simultaneous occurrence with chemotherapy resistance. Hence, antiangiogenesis is still effective after progression on bevacizumab plus chemotherapy. ${ }^{77}$ Indeed, preclinical data indicated that even if alternative angiogenesis pathways emerged, VEGF still plays a vital role in facilitating tumor angiogenesis throughout tumor progression. ${ }^{78}$ Sustained exposure to anti-VEGF mAb achieves tumor regression and extends survival. ${ }^{79,80}$

Two observational cohort studies from BRiTE and ARIES showed that continued bevacizumab beyond first-line progression notably increased the OS compared to chemotherapy alone. ${ }^{81-83}$ This trend is further confirmed in two controlled randomized studies. In the ML18147 trial, maintenance of bevacizumab plus second-line chemotherapy beyond firstline bevacizumab progression had potential clinical benefits in patients with mCRC. The results demonstrated that continuation of bevacizumab with second-line chemotherapy significantly improves PFS (5.7 vs 4.1 months; $P<0.0001$ ) and OS (11.2 vs 9.8 months; $P=0.0062$ ). These benefits were irrespective of KRAS status. The BEBYP trial, similar in design to ML18147, also proved that continued use of bevacizumab during second-line treatment is beneficial. ${ }^{9,10}$

Continued benefits associated with angiogenesis inhibition beyond disease progression can also be observed in other trials. In the phase III study RAISE, the addition of ramucirumab (a human monoclonal antibody that targets VEGFR-2) to second-line treatment improved the outcomes in $\mathrm{mCRC}$ patients beyond first-line bevacizumab progression. ${ }^{84}$ In the VELOUR study, the addition of aflibercept to second-line chemotherapy also improved the survival in bevacizumab pretreated patients. ${ }^{85}$ In third-line treatment or chemo-refractory $\mathrm{mCRC}$, angiogenesis inhibition still has clinical benefits in bevacizumab pretreated patients. Regorafenib and fruquintinib, two VEGFR inhibitors, demonstrated significantly increased OS compared to supportive care, whether patients have received bevacizumab or not. ${ }^{53,86}$

In summary, these data suggest that genome instability leads to the accumulation of more mutations and tumor progression, which may affect the efficacy of anti-EGFR $\mathrm{mAb}$ while an anti-angiogenic agent targets genetically stable endothelial cells, leading to less drug resistance.

\section{Conclusion}

In this review, we overviewed the clinical trials studying the optimal sequence use of anti-EGFR and VEGF mAb in firstline and later lines of treatment in KRAS or RAS WT mCRC, and explored the potential mechanisms that might explain the impaired efficacy of anti-EGFR mAb after progression on first-line bevacizumab and the improved efficacy of antiEGFR $\mathrm{mAb}$ in first-line treatment.

First, we discussed three head-to-head first-line studies comparing anti-EGFR vs VEGF mAb in RAS WT patients. Cetuximab and panitumumab achieved a greater frequency of ETS and DpR that can translate into improved long-term outcomes, supporting the preferential choice for first-line treatment in patients with RAS WT mCRC. However, primary tumor sidedness has a predictive effect for targeted therapy. Anti-EGFR mAb significantly improved survival outcomes in left-sided tumors compared to bevacizumab irrespective of achieving ETS or not. For right-sided tumors, bevacizumab is associated with numerically longer OS compared to antiEGFR mAb. Of note, the survival outcome is similar in both arms for patients with right-sided tumors achieving ETS. Therefore, patients with right-sided tumors achieving ETS might benefit from anti-EGFR mAb in first-line treatment.

Then, we investigated the efficacy of anti-EGFR mAb after progression on first-line bevacizumab in patients with KRAS or RAS WT mCRC. Anti-EGFR mAb did not show an advantage in survival outcomes compared to continuation of bevacizumab in second-line treatment. Bevacizumabinduced hypoxia most likely leads to the poor performance of anti-EGFR mAb in second-line treatment. Indeed, in the COMET and REVERSE trials, cetuximab was more effective in third-line treatment after first-line bevacizumab. A long gap may eliminate the negative effect from hypoxia. Regarding the predictive role of tumor sidedness in treatment sequence, the results are not consistent. In the COMET trial, secondline FOLFOX followed by third-line cetuximab/irinotecan had a better efficacy compared to the reverse sequence in both left- and right-sided tumors. In the REVERSE trials, 
the better outcome was derived from regorafenib followed by cetuximab in patients with left-sided tumors. However, similar benefit was found from this sequence in patients with right-sided tumors. Several retrospective studies also found a benefit from an anti-EGFR mAb and then from bevacizumab treatment sequence based on primary tumor location.

The impacts of sequential therapeutic processes across several treatment lines on the survival kinetics were also explored in FIRE-3. ${ }^{87}$ Markedly different survival kinetics were observed at a change-point of $\sim 22.6$ months in favor of the cetuximab group. The majority of patients completed second- and third-line regimens within the first 2 years, and few patients received effective treatment when OS curves separated. Therefore, optimal sequencing of targeted therapy in first and later lines is important to improve outcomes. This work cannot draw definitive conclusions about the most effective sequence of targeted therapy. We can only speculate that firstline anti-EGFR followed by anti-VEGF is the best sequence in patients with left-sided tumors. Continued use of bevacizumab after progression on first-line bevacizumab is applicable for patients with right-sided tumors, and anti-EGFR mAb may be more appropriate after a third-line chemotherapy regimen or regorafenib. However, for patients with right-sided tumors achieving ETS, the two sequence strategies may be used.

\section{Disclosure}

The authors report no conflicts of interest in this work.

\section{References}

1. Bray F, Ferlay J, Soerjomataram I, et al. Global Cancer statistics 2018: GLOBOCAN estimates of incidence and mortality worldwide for 36 cancers in 185 countries. CA Cancer J Clin. 2018;68(6):394-424.

2. Kopetz S, Chang GJ, Overman MJ, et al. Improved survival in metastatic colorectal cancer is associated with adoption of hepatic resection and improved chemotherapy. J Clin Oncol. 2009;27(22):3677-3683.

3. Heinemann V, von Weikersthal LF, Decker T, et al. FOLFIRI plus cetuximab versus FOLFIRI plus bevacizumab as first-line treatment for patients with metastatic colorectal cancer (FIRE-3): a randomised, open-label, phase 3 trial. Lancet Oncol. 2014;15(10):1065-1075.

4. Schwartzberg LS, Rivera F, Karthaus M, et al. PEAK: a randomized, multicenter phase II study of panitumumab plus modified fluorouracil, leucovorin, and oxaliplatin (mFOLFOX6) or bevacizumab plus mFOLFOX6 in patients with previously untreated, unresectable, wild-type $K R A S$ exon 2 metastatic colorectal cancer. J Clin Oncol. 2014;32(21):2240-2247.

5. National Comprehensive Cancer Network. Clinical practice guidelines in oncology (NCCN guidelines). Colon cancer; 2018. Available from: https://www.nccn.org/. Accessed November 29, 2018.

6. Hurwitz H, Fehrenbacher L, Novotny W, et al. Bevacizumab plus irinotecan, fluorouracil, and leucovorin for metastatic colorectal cancer. N Engl J Med. 2004;350(23):2335-2342.

7. Saltz LB, Clarke S, Díaz-Rubio E, et al. Bevacizumab in combination with oxaliplatin-based chemotherapy as first-line therapy in metastatic colorectal cancer: a randomized phase III study. J Clin Oncol. 2008;26(12):2013-2019.
8. Giantonio BJ, Catalano PJ, Meropol NJ, et al. Bevacizumab in combination with oxaliplatin, fluorouracil, and leucovorin (FOLFOX4) for previously treated metastatic colorectal cancer: results from the Eastern Cooperative Oncology Group study E3200. J Clin Oncol. 2007;25(12):1539-1544.

9. Bennouna J, Sastre J, Arnold D, et al. Continuation of bevacizumab after first progression in metastatic colorectal cancer (ML18147): a randomised phase 3 trial. Lancet Oncol. 2013;14(1):29-37.

10. Masi G, Salvatore L, Boni L, et al. Continuation or reintroduction of bevacizumab beyond progression to first-line therapy in metastatic colorectal cancer: final results of the randomized BEBYP trial. Ann Oncol. 2015;26(4):724-730.

11. Holch JW, Ricard I, Stintzing S, Modest DP, Heinemann V. The relevance of primary tumour location in patients with metastatic colorectal cancer: a meta-analysis of first-line clinical trials. Eur J Cancer. 2017;70:87-98.

12. Arnold D, Lueza B, Douillard JY, et al. Prognostic and predictive value of primary tumour side in patients with RAS wild-type metastatic colorectal cancer treated with chemotherapy and EGFR directed antibodies in six randomized trials. Ann Oncol. 2017;28(8):1713-1729.

13. Boeckx N, Janssens K, van Camp G, et al. The predictive value of primary tumor location in patients with metastatic colorectal cancer: a systematic review. Crit Rev Oncol Hematol. 2018;121:1-10.

14. Tournigand C, André T, Achille E, et al. FOLFIRI followed by FOLFOX6 or the reverse sequence in advanced colorectal cancer: a randomized GERCOR study. J Clin Oncol. 2004;22(2):229-237.

15. Grothey A, Sargent D, Goldberg RM, Schmoll HJ. Survival of patients with advanced colorectal cancer improves with the availability of fluorouracil-leucovorin, irinotecan, and oxaliplatin in the course of treatment. J Clin Oncol. 2004;22(7):1209-1214.

16. Stintzing S, Modest DP, Rossius L, et al. FOLFIRI plus cetuximab versus FOLFIRI plus bevacizumab for metastatic colorectal cancer (FIRE-3): a post-hoc analysis of tumour dynamics in the final RAS wild-type subgroup of this randomised open-label phase 3 trial. Lancet Oncol. 2016;17(10):1426-1434.

17. Venook AP, Niedzwiecki D, Lenz HJ, et al. Effect of first-line chemotherapy combined with cetuximab or bevacizumab on overall survival in patients with $K R A S$ wild-type advanced or metastatic colorectal cancer. JAMA. 2017;317(23):2392-2401.

18. Hecht JR, Cohn A, Dakhil S, et al. SPIRITT: a randomized, multicenter, phase II study of panitumumab with FOLFIRI and bevacizumab with FOLFIRI as second-line treatment in patients with unresectable wild type KRAS metastatic colorectal cancer. Clin Colorectal Cancer. 2015;14(2):72-80.

19. Bennouna J, Hiret S, Bertaut A, et al. Continuation of bevacizumab vs cetuximab plus chemotherapy after first progression in KRAS wild-type metastatic colorectal cancer: the UNICANCER PRODIGE18 randomized clinical trial. JAMA Oncol. 2019;5(1):83-90.

20. Lenz H, Niedzwiecki D, Innocenti F, et al. CALGB/SWOG 80405: phase III trial of irinotecan/5-FU/leucovorin (FOLFIRI) or oxaliplatin/5-FU/ leucovorin (mFOLFOX6) with bevacizumab (BV) or cetuximab (CET) for patients (pts) with expanded ras analyses untreated metastatic adenocarcinoma of the colon or rectum (mCRC). Ann Oncol. 2014;25(Suppl 4):Abstract 5010.

21. Rivera F, Karthaus M, Hecht JR, et al. Final analysis of the randomised PEAK trial: overall survival and tumour responses during first-line treatment with mFOLFOX6 plus either panitumumab or bevacizumab in patients with metastatic colorectal carcinoma. Int J Colorectal Dis. 2017;32(8):1179-1190.

22. Holch JW, Stintzing S, Held S, et al. Right-sided colorectal cancer (RC): response to first-line chemotherapy in FIRE-3 (AIO KRK-0306) with focus on early tumor shrinkage (ETS) and depth of response (DpR). J Clin Oncol. 2017;35(15 Suppl):3586-3586.

23. Peeters M, Price T, Taieb J, et al. Relationships between tumour response and primary tumour location, and predictors of long-term survival, in patients with RAS wild-type metastatic colorectal cancer receiving first-line panitumumab therapy: retrospective analyses of the PRIME and PEAK clinical trials. Br J Cancer. 2018;119(3):303-312. 
24. Norguet E, Dahan L, Gaudart J, et al. Cetuximab after bevacizumab in metastatic colorectal cancer: is it the best sequence? Dig Liver Dis. 2011;43(11):917-919.

25. Derangère V, Fumet JD, Boidot R, et al. Does bevacizumab impact anti-EGFR therapy efficacy in metastatic colorectal cancer? Oncotarget. 2016;7(8):9309-9321.

26. Sato Y, Matsusaka S, Suenaga M, Shinozaki E, Mizunuma N. Cetuximab could be more effective without prior bevacizumab treatment in metastatic colorectal cancer patients. Onco Targets Ther. 2015;8: 3329-3336.

27. Taniguchi H, Komori A, Narita Y, et al. A short interval between bevacizumab and anti-epithelial growth factor receptor therapy interferes with efficacy of subsequent anti-EGFR therapy for refractory colorectal cancer. Jpn J Clin Oncol. 2016;46(3):228-233.

28. Peeters M, Price TJ, Cervantes A, et al. Final results from a randomized phase 3 study of FOLFIRI \pm panitumumab for second-line treatment of metastatic colorectal cancer. Ann Oncol. 2014;25(1):107-116.

29. Deng Y, Cai Y, Lin J, et al. Survival of patients with KRAS wild-type metastatic colorectal cancer is identical after sequential treatment with cetuximab and bevacizumab regardless of the sequence - A retrospective single-center study. Gastroenterol Rep. 2015;3(4):339-343.

30. Price TJ, Hardingham J, Karapetis C, et al. 524P Bevacizumab first line and impact on subsequent anti-EGFR activity. Ann Oncol. 2017; 28(Suppl 5).

31. Buchler T, Chloupkova R, Poprach A, et al. Sequential therapy with bevacizumab and epidermal growth factor receptor-directed agents for metastatic colorectal carcinoma: a retrospective, registry-based analysis. Ann Oncol. 2017;28(Suppl 5).

32. Burge M, Semira C, Lee B, et al. Previous bevacizumab and efficacy of later anti-epidermal growth factor receptor antibodies in metastatic colorectal cancer: results from a large international registry. Clin Colorectal Cancer. 2018;17(3):e593-e599.

33. Modest DP, Stintzing S, von Weikersthal LF, et al. Impact of subsequent therapies on outcome of the FIRE-3/AIO KRK0306 trial: first-line therapy with FOLFIRI plus cetuximab or bevacizumab in patients with KRAS wild-type tumors in metastatic colorectal cancer. J Clin Oncol. 2015;33(32):3718-3726.

34. Modest DP, Stintzing S, von Weikersthal LF, et al. Exploring the effect of primary tumor sidedness on therapeutic efficacy across treatment lines in patients with metastatic colorectal cancer: analysis of FIRE-3 (AIOKRK0306). Oncotarget. 2017;8(62):105749-105760.

35. Peeters M, Forget F, Karthaus M, et al. Exploratory pooled analysis evaluating the effect of sequence of biological therapies on overall survival in patients with $R A S$ wild-type metastatic colorectal carcinoma. ESMO Open. 2018;3(2):e000297.

36. Shitara K, Yonesaka K, Denda T, et al. Randomized study of FOLFIRI plus either panitumumab or bevacizumab for wild-type KRAS colorectal cancer-WJOG 6210G. Cancer Sci. 2016;107(12):1843-1850.

37. Cascinu S, Rosati G, Nasti G, et al. Treatment sequence with either irinotecan/cetuximab followed by FOLFOX-4 or the reverse strategy in metastatic colorectal cancer patients progressing after first-line FOLFIRI/bevacizumab: an Italian Group for the study of gastrointestinal cancer phase III, randomised trial comparing two sequences of therapy in colorectal metastatic patients. Eur J Cancer. 2017;83:106-115.

38. Shitara K, Yamanaka T, Denda T, et al. Reverce: randomized phase II study of regorafenib followed by cetuximab versus the reverse sequence for metastatic colorectal cancer patients previously treated with fluoropyrimidine, oxaliplatin, and irinotecan. J Clin Oncol. 2018;36(4 Suppl):Abstract 557.

39. Pàez-Ribes M, Allen E, Hudock J, et al. Antiangiogenic therapy elicits malignant progression of tumors to increased local invasion and distant metastasis. Cancer Cell. 2009;15(3):220-231.

40. Jain RK. Normalization of tumor vasculature: an emerging concept in antiangiogenic therapy. Science. 2005;307(5706):58-62.

41. Jain RK. Normalizing tumor microenvironment to treat cancer: Bench to bedside to biomarkers. J Clin Oncol. 2013;31(17):2205-2218.
42. Mimeault M, Batra SK. Hypoxia-inducing factors as master regulators of stemness properties and altered metabolism of cancer- and metastasisinitiating cells. J Cell Mol Med. 2013;17(1):30-54.

43. Mésange P, Poindessous V, Sabbah M, et al. Intrinsic bevacizumab resistance is associated with prolonged activation of autocrine VEGF signaling and hypoxia tolerance in colorectal cancer cells and can be overcome by nintedanib, a small molecule angiokinase inhibitor. Oncotarget. 2014;5(13):4709-4721.

44. Bos R, van Diest PJ, de Jong JS, et al. Hypoxia-inducible factor-1alpha is associated with angiogenesis, and expression of bFGF, PDGF-BB, and EGFR in invasive breast cancer. Histopathology. 2005;46(1):31-36.

45. Swinson DE, O'Byrne KJ. Interactions between hypoxia and epidermal growth factor receptor in non-small-cell lung cancer. Clin Lung Cancer. 2006;7(4):250-256.

46. Setty BA, Pillay Smiley N, Pool CM, Jin Y, Liu Y, Nelin LD. Hypoxiainduced proliferation of HeLa cells depends on epidermal growth factor receptor-mediated arginase II induction. Physiol Rep. 2017;5(6):e13175.

47. Seton-Rogers S. Hypoxia: new connections. Nature Rev Cancer. 2012;12:320-321.

48. Keith B, Johnson RS, Simon MC. HIF1 $\alpha$ and HIF2 $\alpha$ : sibling rivalry in hypoxic tumour growth and progression. Nat Rev Cancer. 2011;12(1):9-22.

49. Wouters A, Boeckx C, Vermorken JB, van den Weyngaert D, Peeters $\mathrm{M}$, Lardon $\mathrm{F}$. The intriguing interplay between therapies targeting the epidermal growth factor receptor, the hypoxic microenvironment and hypoxia-inducible factors. Curr Pharm Des. 2013;19(5):907-917.

50. Wang Y, Lei F, Rong W, Zeng Q, Sun W. Positive feedback between oncogenic KRas and HIF- $1 \alpha$ confers drug resistance in colorectal cancer. Onco Targets Ther. 2015;8:1229-1237.

51. Zeng M, Kikuchi H, Pino MS, Chung DC. Hypoxia activates the K-ras proto-oncogene to stimulate angiogenesis and inhibit apoptosis in colon cancer cells. PLoS One. 2010;5(6):e10966.

52. Taniguchi H, Baba Y, Sagiya Y, et al. Biologic response of colorectal cancer xenograft tumors to sequential treatment with panitumumab and bevacizumab. Neoplasia. 2018;20(7):668-677.

53. Grothey A, van Cutsem E, Sobrero A, et al. Regorafenib monotherapy for previously treated metastatic colorectal cancer (CORRECT): an international, multicentre, randomised, placebo-controlled, phase 3 trial. Lancet. 2013;381(9863):303-312.

54. Willett CG, Boucher Y, di Tomaso E, et al. Direct evidence that the VEGF-specific antibody bevacizumab has antivascular effects in human rectal cancer. Nat Med. 2004;10(2):145-147.

55. Willett CG, Boucher Y, Duda DG, et al. Surrogate markers for antiangiogenic therapy and dose-limiting toxicities for bevacizumab with radiation and chemotherapy: continued experience of a phase I trial in rectal cancer patients. J Clin Oncol. 2005;23(31):8136-8139.

56. Navarro P, Bueno MJ, Zagorac I, et al. Targeting tumor mitochondrial metabolism overcomes resistance to Antiangiogenics. Cell Rep. 2016;15(12):2705-2718.

57. Elez E, Argilés G, Tabernero J. First-line treatment of metastatic colorectal cancer: interpreting FIRE-3, PEAK, and CALGB/SWOG 80405. Curr Treat Options Oncol. 2015;16(11):52.

58. Khattak MA, Martin H, Davidson A, Phillips M. Role of first-line antiepidermal growth factor receptor therapy compared with anti-vascular endothelial growth factor therapy in advanced colorectal cancer: a meta-analysis of randomized clinical trials. Clin Colorectal Cancer. 2015;14(2):81-90.

59. Heinemann V, Rivera F, O'Neil BH, et al. A study-level meta-analysis of efficacy data from head-to-head first-line trials of epidermal growth factor receptor inhibitors versus bevacizumab in patients with RAS wild-type metastatic colorectal cancer. Eur J Cancer. 2016;67:11-20.

60. Venook AP, Tabernero J. Progression-free survival: Helpful biomarker or clinically meaningless end point? J Clin Oncol. 2015;33(1):4-6.

61. Suzuki C, Blomqvist L, Sundin A, et al. The initial change in tumor size predicts response and survival in patients with metastatic colorectal cancer treated with combination chemotherapy. Ann Oncol. 2012;23(4):948-954. 
62. Giessen C, Laubender RP, Fischer von Weikersthal L, et al. Early tumor shrinkage in metastatic colorectal cancer: retrospective analysis from an irinotecan-based randomized first-line trial. Cancer Sci. 2013;104(6):718-724.

63. Modest DP, Laubender RP, Stintzing S, et al. Early tumor shrinkage in patients with metastatic colorectal cancer receiving first-line treatment with cetuximab combined with either CAPIRI or CAPOX: an analysis of the German AIO KRK 0104 trial. Acta Oncol. 2013;52(5):956-962.

64. Douillard JY, Siena S, Peeters M, Koukakis R, Terwey JH, Tabernero J. Impact of early tumour shrinkage and resection on outcomes in patients with wild-type RAS metastatic colorectal cancer. Eur J Cancer. 2015;51(10):1231-1242.

65. Petrelli F, Pietrantonio F, Cremolini C, et al. Early tumour shrinkage as a prognostic factor and surrogate end-point in colorectal cancer: a systematic review and pooled-analysis. Eur J Cancer. 2015;51(7):800-807.

66. Heinemann V, Stintzing S, Modest DP, et al. Early tumour shrinkage (ETS) and depth of response (DpR) in the treatment of patients with metastatic colorectal cancer (mCRC). Eur J Cancer. 2015;51(14):1927-1936.

67. Piessevaux H, Buyse M, Schlichting M, et al. Use of early tumor shrinkage to predict long-term outcome in metastatic colorectal cancer treated with cetuximab. J Clin Oncol. 2013;31(30):3764-3775.

68. Mansmann UR, Sartorius U, Laubender RP, et al. Deepness of response: a quantitative analysis of its impact on post-progression survival time after first-line treatment in patients with mCRC. J Clin Oncol. 2013;31(4 Suppl):Abstr 427.

69. Taieb J, Rivera F, Siena S, et al. Exploratory analyses assessing the impact of early tumour shrinkage and depth of response on survival outcomes in patients with RAS wild-type metastatic colorectal cancer receiving treatment in three randomised panitumumab trials. J Cancer Res Clin Oncol. 2018;144(2):321-335.

70. Cremolini C, Loupakis F, Antoniotti C, et al. Early tumor shrinkage and depth of response predict long-term outcome in metastatic colorectal cancer patients treated with first-line chemotherapy plus bevacizumab: results from phase III tribe trial by the Gruppo Oncologico del Nord Ovest. Ann Oncol. 2015;26(6):1188-1194.

71. Loupakis F, Cremolini C, Masi G, et al. Initial therapy with FOLFOXIRI and bevacizumab for metastatic colorectal cancer. $N$ Engl J Med. 2014;371(17):1609-1618.

72. Hanahan D, Weinberg RA. Hallmarks of cancer: the next generation. Cell. 2011;144(5):646-674.

73. Misale S, di Nicolantonio F, Sartore-Bianchi A, Siena S, Bardelli A. Resistance to anti-EGFR therapy in colorectal cancer: from heterogeneity to convergent evolution. Cancer Discov. 2014;4(11):1269-1280.

74. Seymour MT, Brown SR, Middleton G, et al. Panitumumab and irinotecan versus irinotecan alone for patients with KRAS wild-type, fluorouracil-resistant advanced colorectal cancer (Piccolo): a prospectively stratified randomised trial. Lancet Oncol. 2013;14(8):749-759.
75. Misale S, Yaeger R, Hobor S, et al. Emergence of KRAS mutations and acquired resistance to anti-EGFR therapy in colorectal cancer. Nature. 2012;486(7404):532-536.

76. Hc W, Pc L. Proteins expressed on tumor endothelial cells as potential targets for anti-angiogenic therapy. J Cancer Molec. 2008;4:17-22.

77. Giantonio BJ. Targeted therapies: Goldie-Coldman and bevacizumab beyond disease progression. Nat Rev Clin Oncol. 2009;6(6):311-312.

78. Bergers G, Benjamin LE. Tumorigenesis and the angiogenic switch. Nat Rev Cancer. 2003;3(6):401-410.

79. Bagri A, Berry L, Gunter B, et al. Effects of anti-VEGF treatment duration on tumor growth, tumor regrowth, and treatment efficacy. Clin Cancer Res. 2010;16(15):3887-3900.

80. Klement G, Baruchel S, Rak J, et al. Continuous low-dose therapy with vinblastine and VEGF receptor-2 antibody induces sustained tumor regression without overt toxicity. J Clin Invest. 2000;105(8):R15-R24.

81. Cohn AL, Bekaii-Saab T, Bendell JC, et al. Clinical outcomes in bevacizumab (BV)-treated patients (pts) with metastatic colorectal cancer (mCRC): results from ARIES observational cohort study (OCS) and confirmation of BRiTE data on BV beyond progression (BBP). J Clin Oncol. 2010;28(15):3596-3596.

82. Grothey A, Sugrue MM, Purdie DM, et al. Bevacizumab beyond first progression is associated with prolonged overall survival in metastatic colorectal cancer: results from a large observational cohort Study (BRiTE). J Clin Oncol. 2008;26(33):5326-5334.

83. Hurwitz HI, Bekaii-Saab TS, Bendell JC, et al. Safety and effectiveness of bevacizumab treatment for metastatic colorectal cancer: final results from the Avastin ${ }^{\circ}$ registry - investigation of effectiveness and safety (ARIES) observational cohort study. Clin Oncol. 2014;26(6): 323-332.

84. Tabernero J, Yoshino T, Cohn AL, et al. Ramucirumab versus placebo in combination with second-line FOLFIRI in patients with metastatic colorectal carcinoma that progressed during or after first-line therapy with bevacizumab, oxaliplatin, and a fluoropyrimidine (RAISE): a randomised, double-blind, multicentre, phase 3 study. Lancet Oncol. 2015;16(5):499-508.

85. van Cutsem E, Tabernero J, Lakomy R, et al. Addition of aflibercept to fluorouracil, leucovorin, and irinotecan improves survival in a phase III randomized trial in patients with metastatic colorectal cancer previously treated with an oxaliplatin-based regimen. J Clin Oncol. 2012;30(28):3499-3506.

86. Li J, Qin S, Xu RH, et al. Effect of Fruquintinib vs placebo on overall survival in patients with previously treated metastatic colorectal cancer: the FRESCO randomized clinical trial. JAMA. 2018;319(24): 2486-2496.

87. Modest DP, Ricard I, Stintzing S, et al. Evaluation of survival across several treatment lines in metastatic colorectal cancer: analysis of the FIRE-3 trial (AIO KRK0306). Eur J Cancer. 2017;84:262-269.
Cancer Management and Research

\section{Publish your work in this journal}

Cancer Management and Research is an international, peer-reviewed open access journal focusing on cancer research and the optimal use of preventative and integrated treatment interventions to achieve improved outcomes, enhanced survival and quality of life for the cancer patient. The manuscript management system is completely online and includes

\section{Dovepress}

a very quick and fair peer-review system, which is all easy to use. Visit http://www.dovepress.com/testimonials.php to read real quotes from published authors. 